\title{
Small-Sized MOF-Constructed Multifunctional Diagnosis and Therapy \\ Platform for Tumor
}

Chao Wang, ${ }^{a b}$ Xiaodan Jia, ${ }^{a}$ Wenyao Zhen ${ }^{a b}$, Mengchao Zhang*c and Xiue Jiang*ab

a. State Key Laboratory of Electroanalytical Chemistry. Changchun Institute of Applied Chemistry,

Chinese Academy of Sciences, Changchun, Jilin 130022, P. R. China

b. University of Science and Technology of China, Hefei, Anhui 230029, P. R. China

c. The Department of Radiology, China-Japan Union Hospital of Jilin University, Changchun, Jilin 130022, P. R. China

*E-mail: xiuejiang@ciac.ac.cn. Tel: +86-431-85262069 (X.J.).

*E-mail: zhangmengchao@jlu.edu.cn (M.Z).

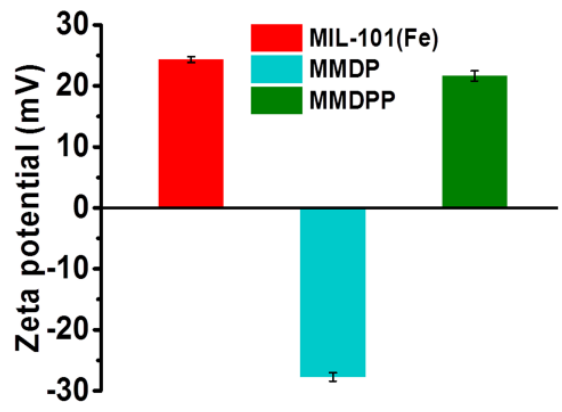

Figure S1. Zeta potential of MIL-101(Fe), MOFs-MB-DHA@PLA and MOFs-MB-DHA@PLA @PEG.

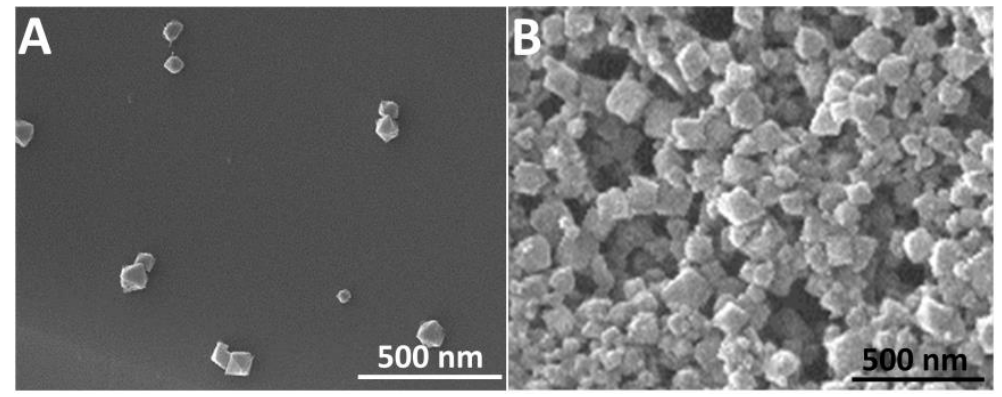

Figure S2. SEM images of MOFs (A) and MMDPP (B). 

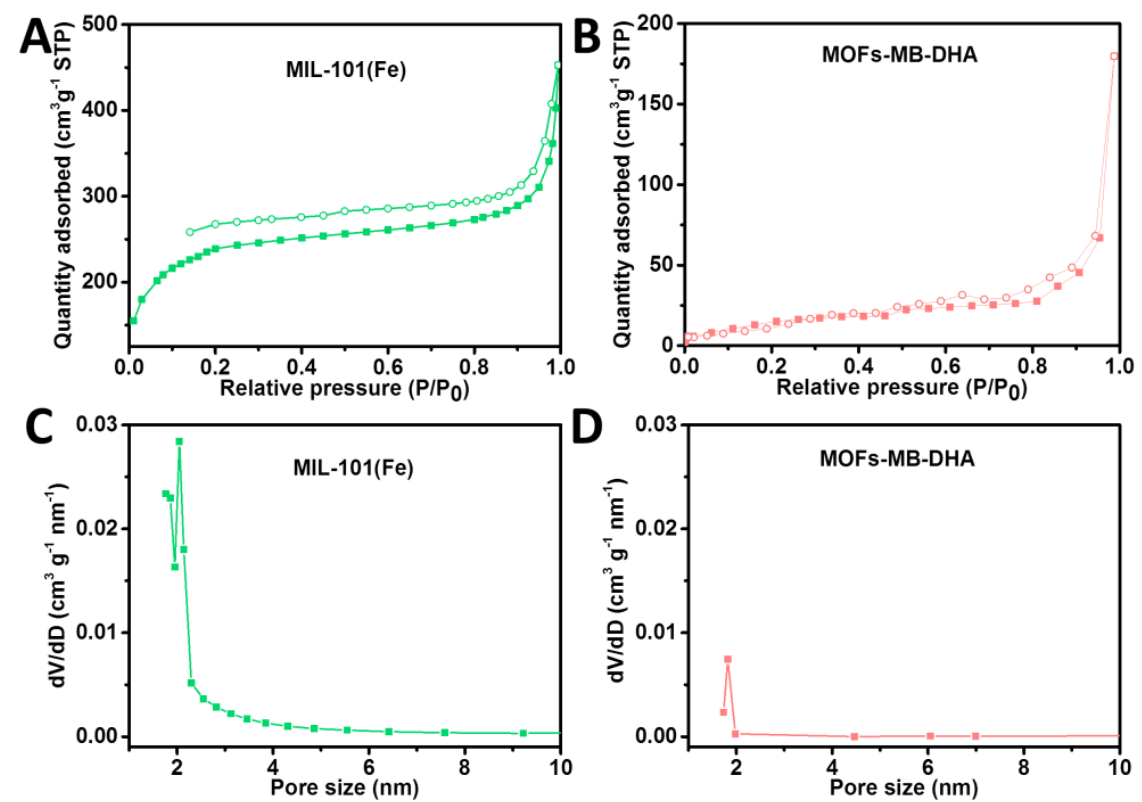

Figure S3. The $\mathrm{N}_{2}$ isotherm of MIL-101(Fe) and MOFs-MB-DHA at 77K ( $\mathrm{A}$ and $\mathrm{B}$ ) and pore size distribution of MIL-101(Fe) and MOFs-MB-DHA derived from $\mathrm{N}_{2}$ isotherm data ( $C$ and D).

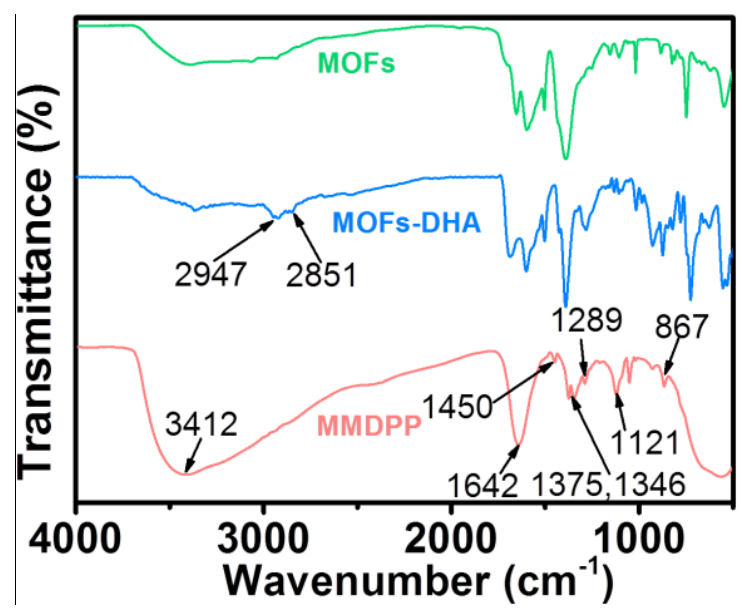

Figure S4. The FTIR spectra of MOFs, MOFs-DHA, and MMDPP.

The spectrum of MOFs was the same as the reported literatures, indicating the successful synthesis of MIL-101(Fe). For the spectrum of MOFs-DHA, the spectral band appeared at about $2947 \mathrm{~cm}^{-1}$ and $2851 \mathrm{~cm}^{-1}$ for strong $\mathrm{CH}_{3}$ and $\mathrm{CH}_{2}$ absorption of DHA, indicating the successful loading of DHA into the pores of MOFs. The MMDPP showed visible difference from single MOFs. More precisely, for the spectrum of MMDPP, the spectral band appeared at about $3412 \mathrm{~cm}^{-1}$ for $\mathrm{N}-\mathrm{H}$ and $\mathrm{O}-\mathrm{H}$ stretching vibrations of $\mathrm{PEG}, 1346 \mathrm{~cm}^{-1}$ for $\mathrm{C}-\mathrm{H}$ bending vibrations of PEG, 1289 $\mathrm{cm}^{-1}$ for alcoholic O-H stretching vibrations of PEG, and $1121 \mathrm{~cm}^{-1}$ for $\mathrm{C}-\mathrm{O}-\mathrm{H}$ bending of PEG; the spectral band appeared at about $1642 \mathrm{~cm}^{-1}$ for $\mathrm{C}=\mathrm{O}$ bond of PLA, $1450 \mathrm{~cm}^{-1}$ for asymmetric $\mathrm{C}-\mathrm{H}$ deformation vibrations of PLA, $1375 \mathrm{~cm}^{-1} \mathrm{CH}_{3}$ bending of PLA, and $867 \mathrm{~cm}^{-1}$ for amorphous phase of PLA. These further suggested the complete coating of PLA and PEG onto the MOFs. 

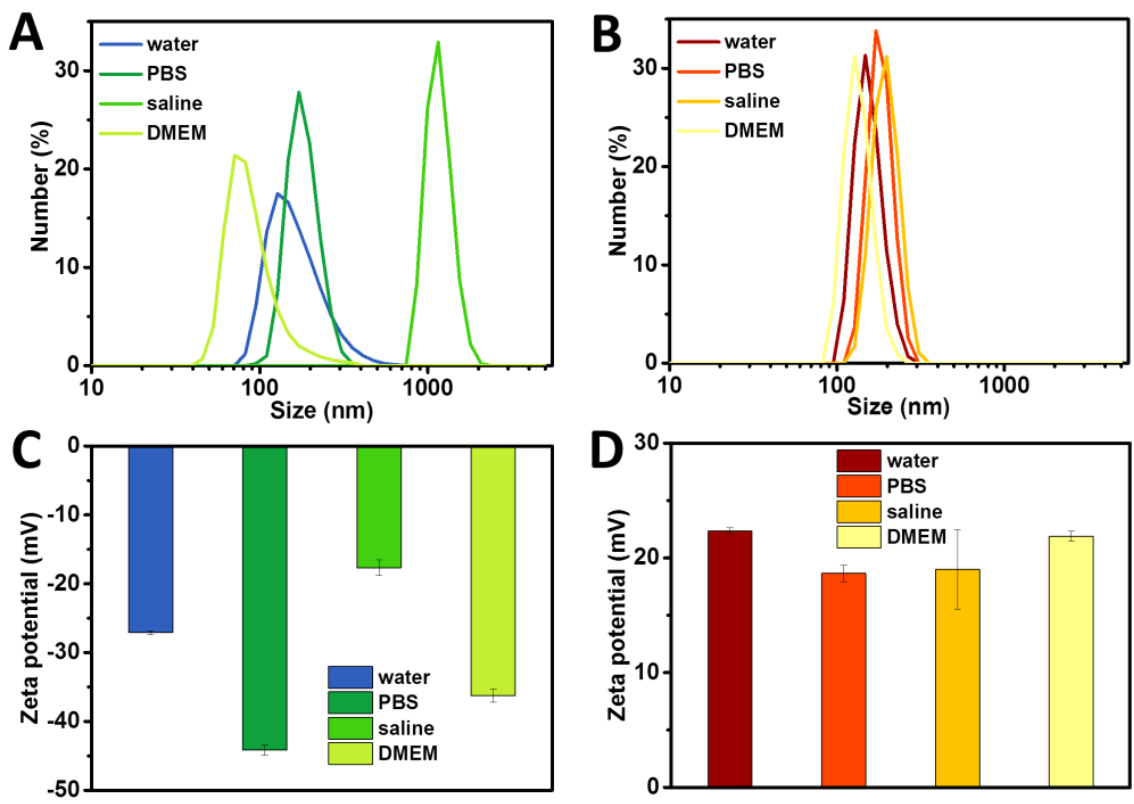

Figure S5. The DLS measurements of MMDP and MMDPP after different treatments for 5 days (A and $B$ ) and corresponding zeta potential of MMDP and MMDPP ( $C$ and $D)$.
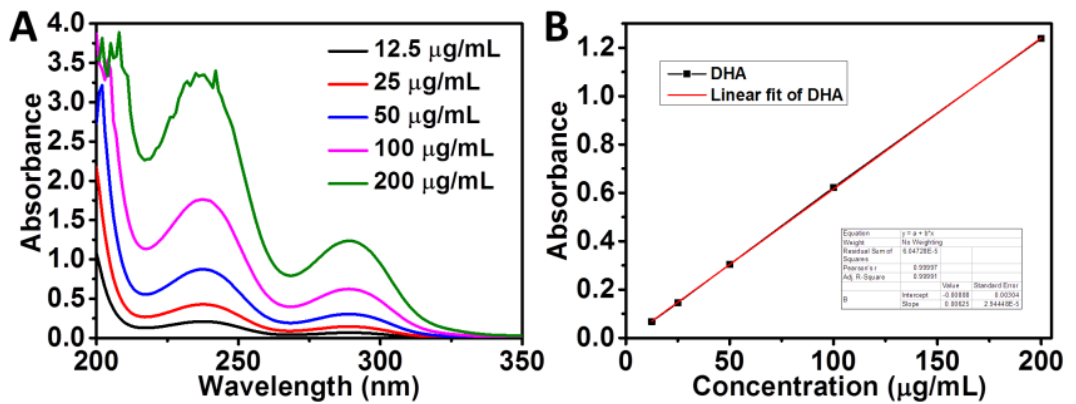

Figure S6. (A) The UV-vis absorbance spectra of reacted mixture of $\mathrm{NaOH}$ and DHA with different concentrations. (B) The standard curve of DHA calculated by the absorbance at $289 \mathrm{~nm}$ through (A).
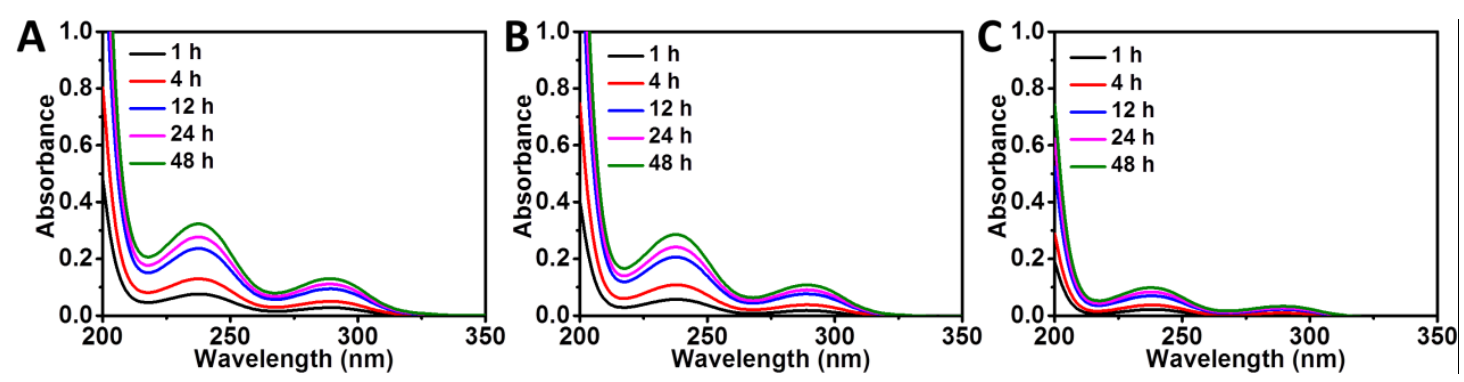

Figure S7. The absorbance spectra of reacted mixture of $\mathrm{NaOH}$ and DHA released from MDPP at different times under pH $5.5+\mathrm{k}(\mathrm{A}), \mathrm{pH} 5.5$ (B) and pH 7.4 (C). 

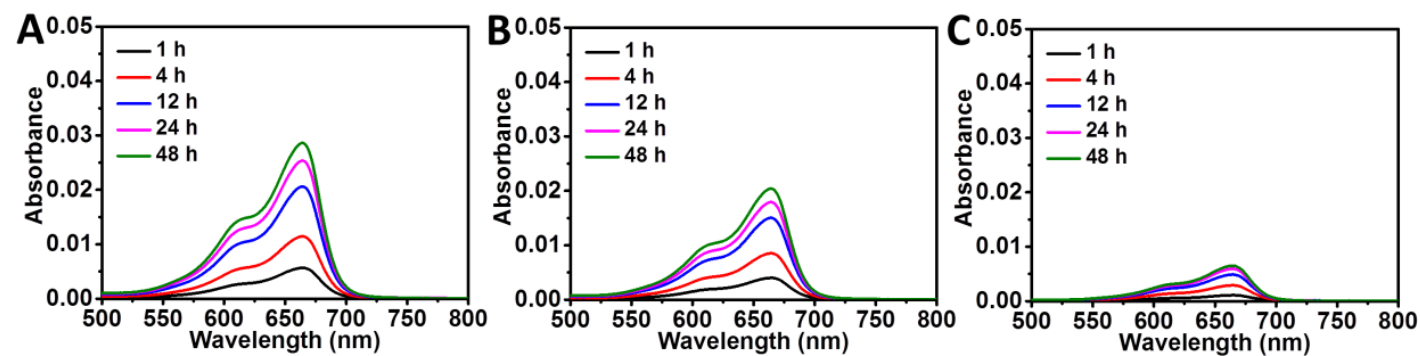

Figure S8. The absorbance spectra of MB released from MMPP at different times under $\mathrm{pH} 5.5+$ $\mathrm{k}(\mathrm{A}), \mathrm{pH} 5.5$ (B) and $\mathrm{pH} 7.4$ (C).

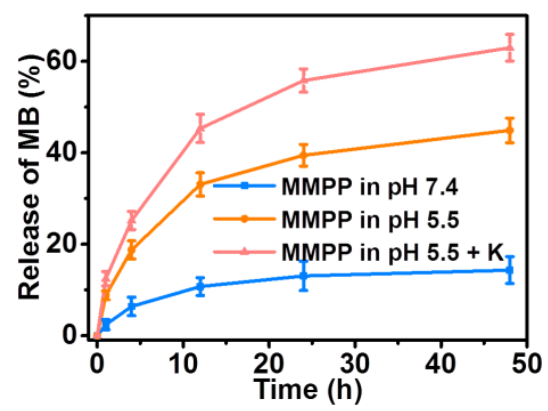

Figure S9. In vitro release profiles of MB-loaded MOFs under different conditions.
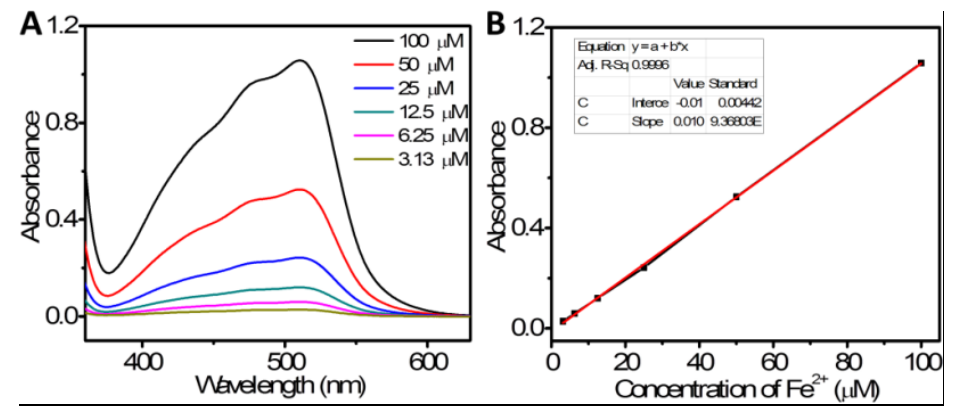

Figure S10. (A) The absorbance spectra of the o-phenanthroline reacted with $\mathrm{Fe}^{3+}$ in different concentrations which has absorbance peak at $509 \mathrm{~nm}$ and (B) the standard curve of it.
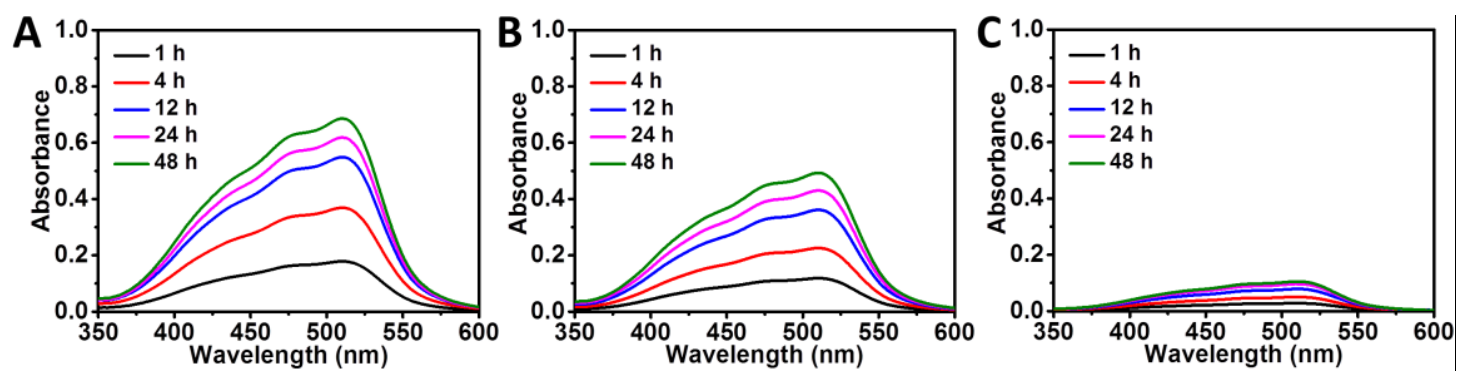

Figure S11. The absorbance spectra of released $\mathrm{Fe}^{3+}$ from MPP reacted with o-phenanthroline at different times under pH $5.5+\mathrm{K}(\mathrm{A}), \mathrm{pH} 5.5$ (B) and pH 7.4 (C). 

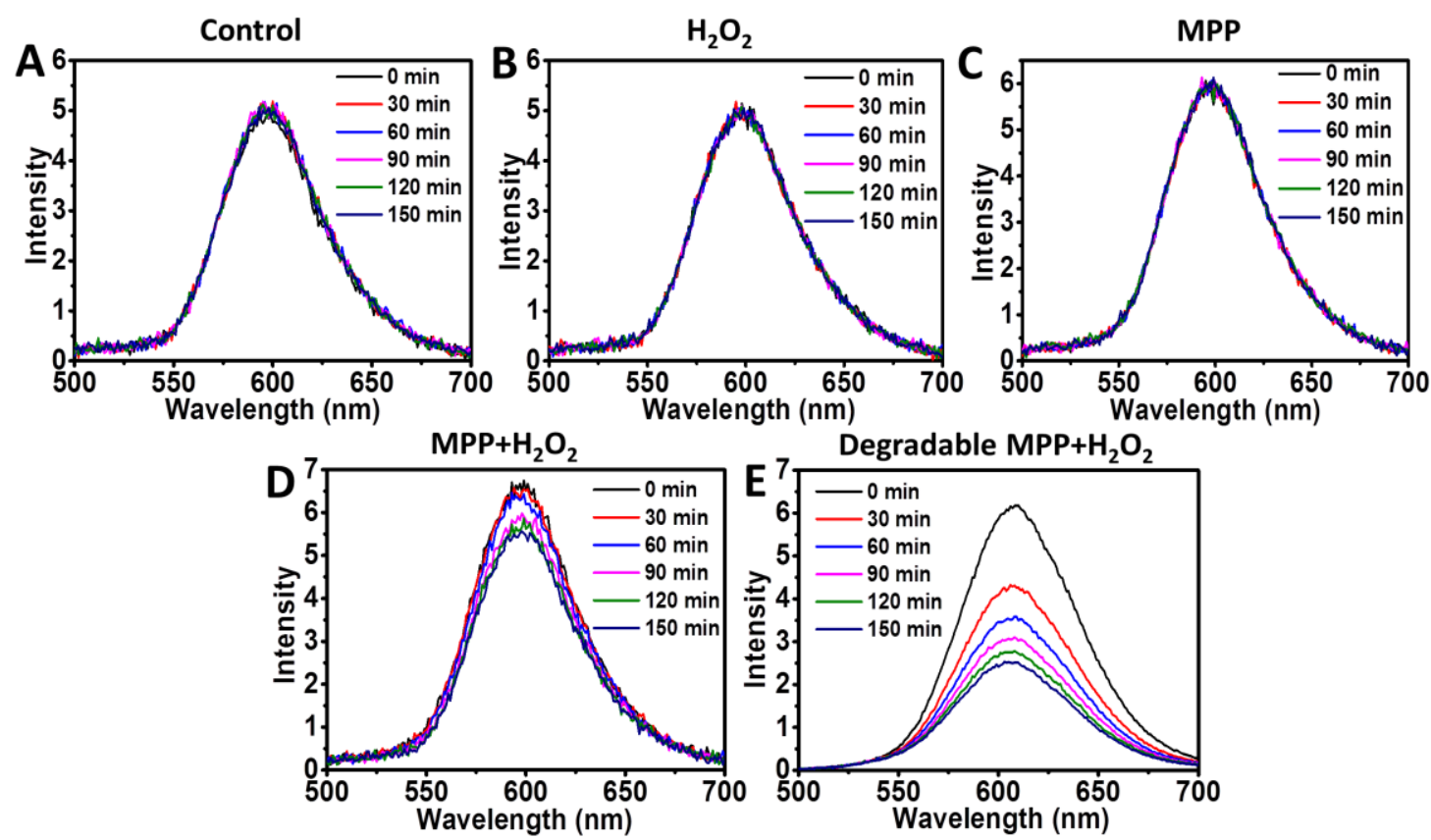

Figure S12. Detection of oxygen consumption by Tris (2,2'-bipyridine) ruthenium dichloride (RDPP) in different groups. The concentration of $\mathrm{H}_{2} \mathrm{O}_{2}$ was $10 \mathrm{mM}$, for MPP, it was $20 \mu \mathrm{gL}^{-1}$. The degradable MPP was pre-cultured in PBS $(\mathrm{pH} 5.5+\mathrm{K})$ for $24 \mathrm{~h}$.
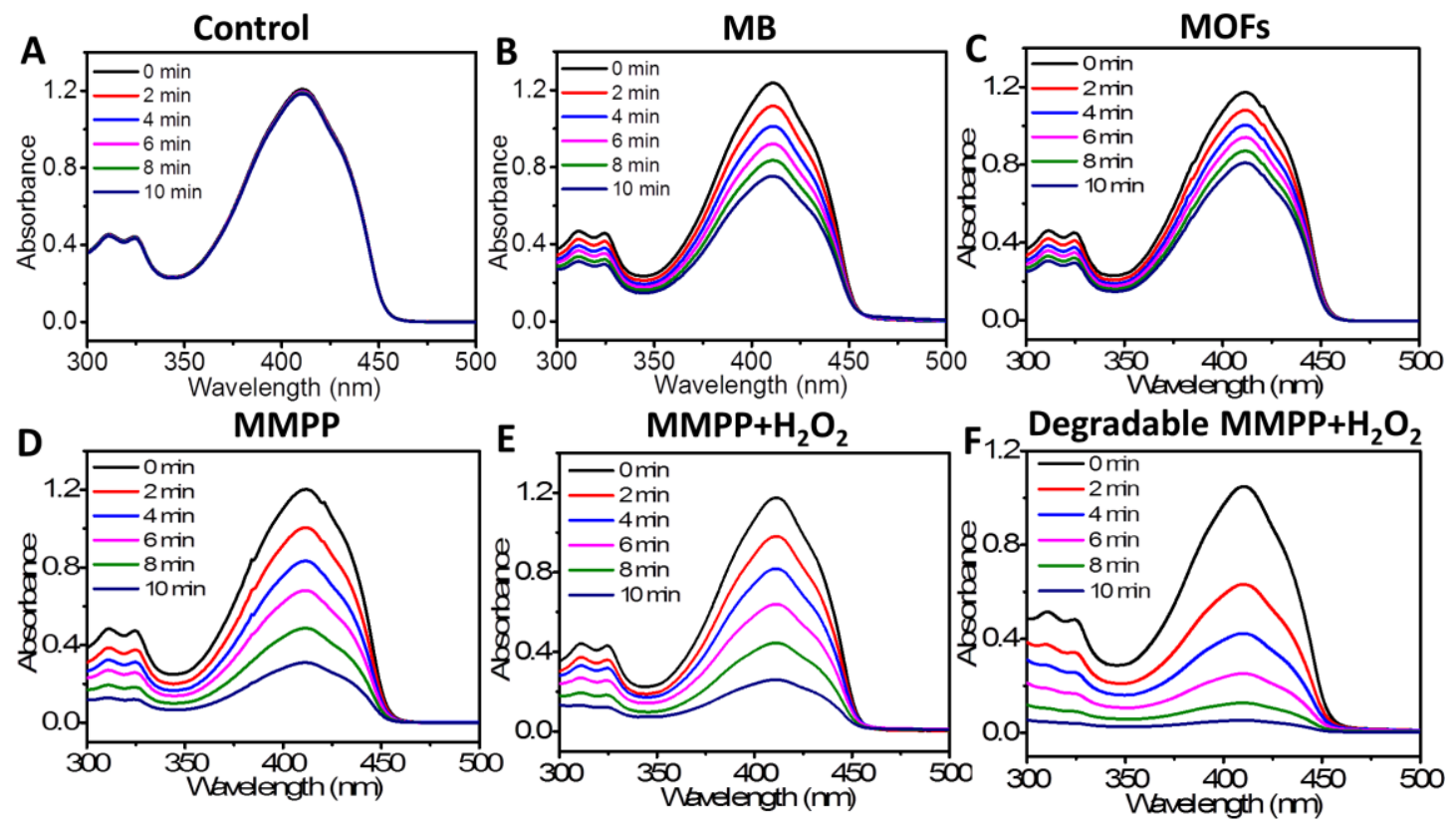

Figure S13. Consumption of DPBF over time due to ${ }^{1} \mathrm{O}_{2}$ generation in different groups of control $(A), M B(B), M O F s(C), M M P P(D), M M P P+\mathrm{H}_{2} \mathrm{O}_{2}(E)$ and degradable MMPP $+\mathrm{H}_{2} \mathrm{O}_{2}(F)$. The concentrations of $\mathrm{MB}$ and $\mathrm{H}_{2} \mathrm{O}_{2}$ were $0.05 \mu \mathrm{g} / \mathrm{mL}$ and $10 \mathrm{mM}$ respectively. It was $100 \mu \mathrm{g} / \mathrm{mL}$ for MOFs and MMPP. Degradable MMPP was pre-cultured in PBS (pH $5.5+\mathrm{K})$ for $24 \mathrm{~h}$. 


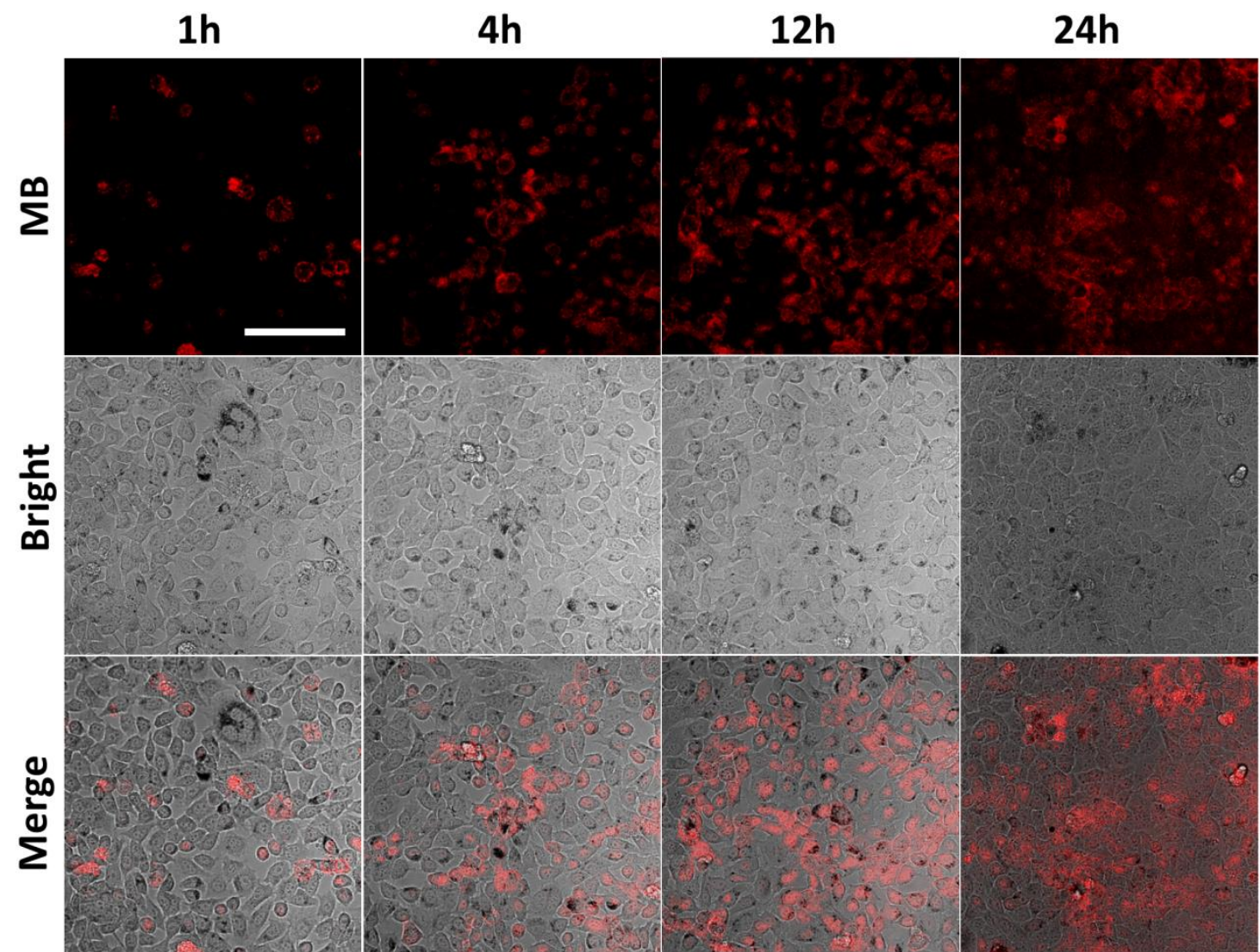

Figure S14. The endocytosis effect of MMDPP in HeLa cells at different time points after incubated with the nanocomposites. The fluorescence was captured from MB (Ex: $635 \mathrm{~nm}, \mathrm{Em}$ : 660-710 nm). Scale bar is $150 \mu \mathrm{m}$.

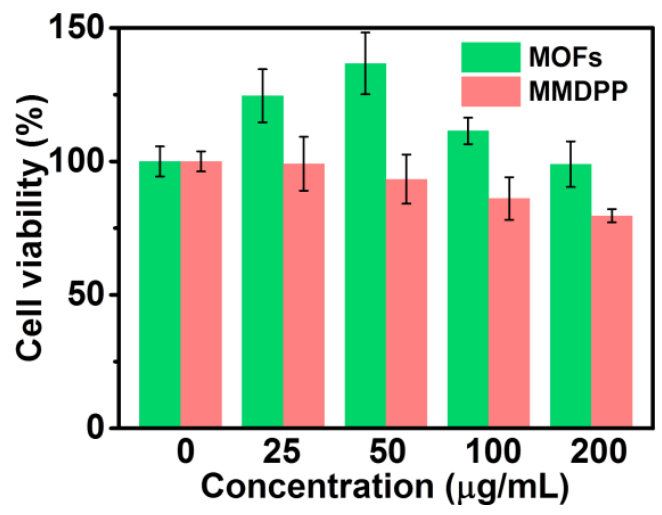

Figure S15. Viability of the L929 cells treated with MOFs or MMDPP at various concentrations.

From the results, the single MOFs didn't show any cytotoxicity to normal cells even the concentration was up-regulated to $200 \mu \mathrm{g} \mathrm{mL}-1$. After cultured with MMDPP $\left(200 \mu \mathrm{g} \mathrm{mL}^{-1}\right)$, the cell viability was reduced slightly (79.6\%), however, it showed significant difference with HeLa cells $(17.6 \%)$, indicating the weak side effect of the material.

The material showed selective cytotoxicity owing to following facts. First, the PLA shell was more easily to degrade in tumor microenvironment owing to the high content of proteinase $\mathrm{K}$, the degradation of PLA further promoted lot release of iron ions and drugs to enhance the 
curative effect. Secondly, the high concentration of $\mathrm{H}_{2} \mathrm{O}_{2}$ in tumor cells further induced high PDT effect owing to abundant supply of oxygen. Thirdly, in the part of PDT, the tumor tissue was irradiated by external laser accurately and this process won't produce any side effect to other tissues. In conclusion, the material showed obvious difference of cytotoxicity between normal cells and tumor cells.

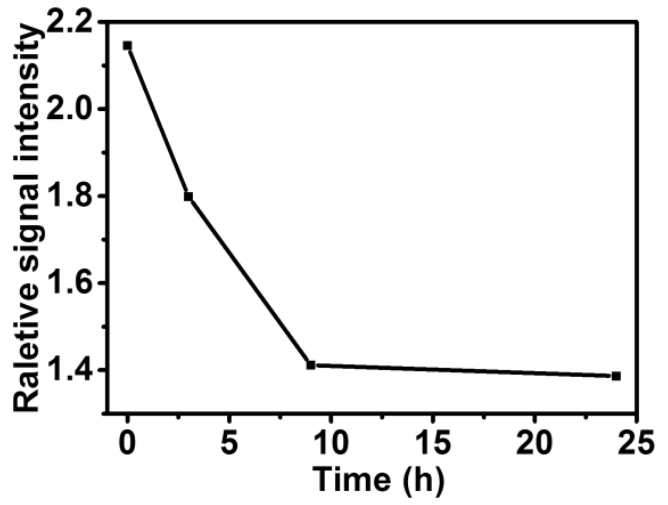

Figure S16. The relative signal intensiy of tumor to normal tissue calculated from the marked region in Figure 4D-4G.

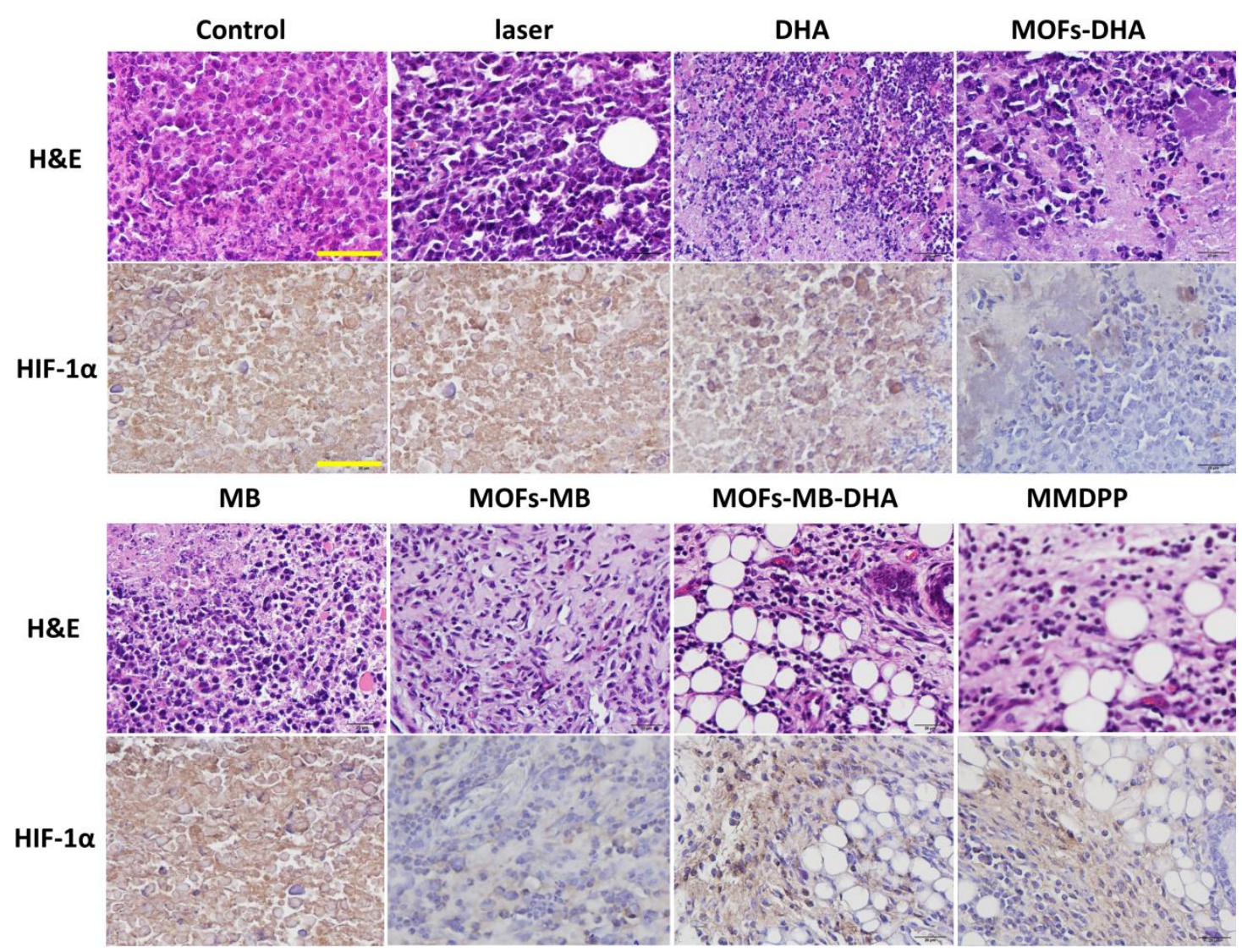

Figure S17. H\&E and HIF-1 $\alpha$ stained tumor slides from U14 tumor bearing mice with several treatments at the 15 th day. Scale bars are $40 \mu \mathrm{m}$. 


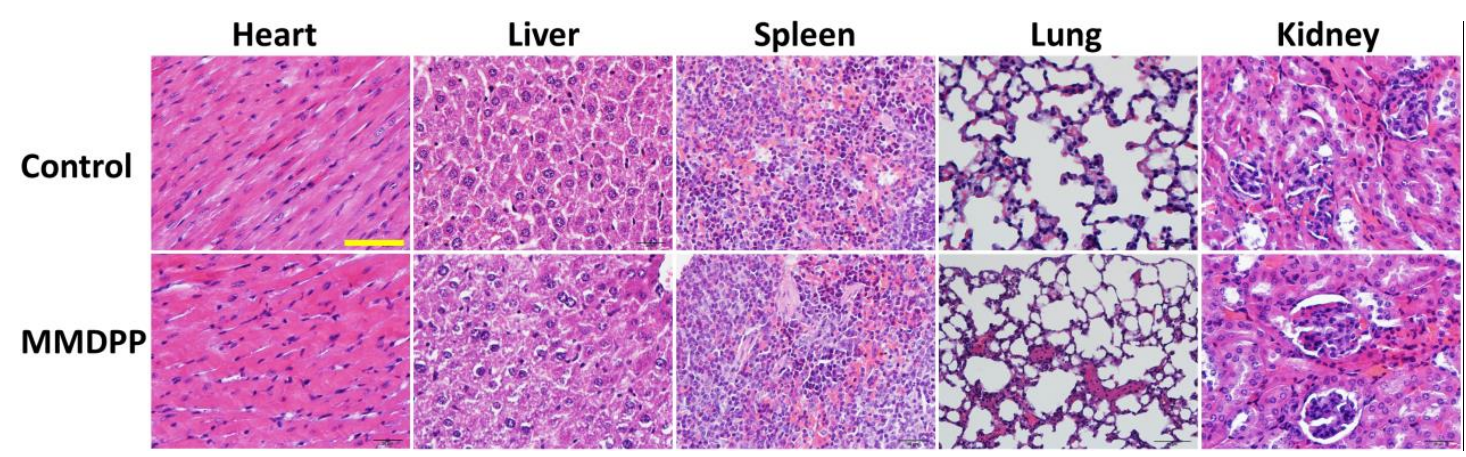

Figure S18. H\&E stained tissue slides from U14 tumor bearing mice of control group and MMDPP group at the 15 th day. Scale bars are $40 \mu \mathrm{m}$.

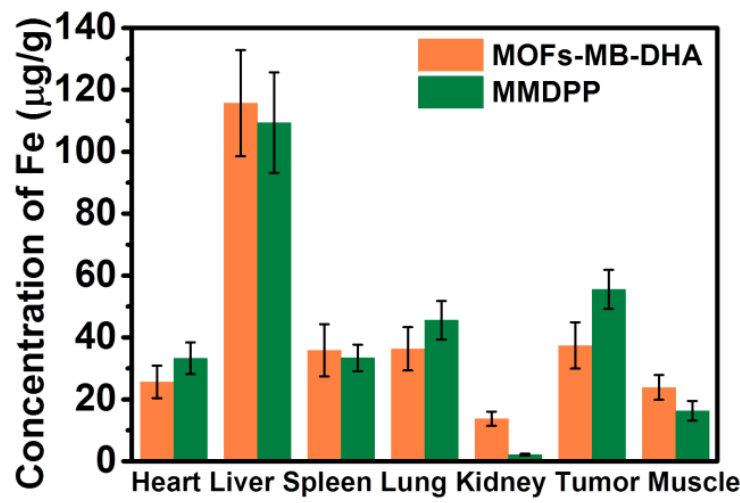

Figure S19. Biodistribution of MOFs-MB-DHA and MMDPP in major organs and tumor tissue at 24 hours post intravenous injection of nanocomposites evaluated by the content of Fe ion measuring with ICP.

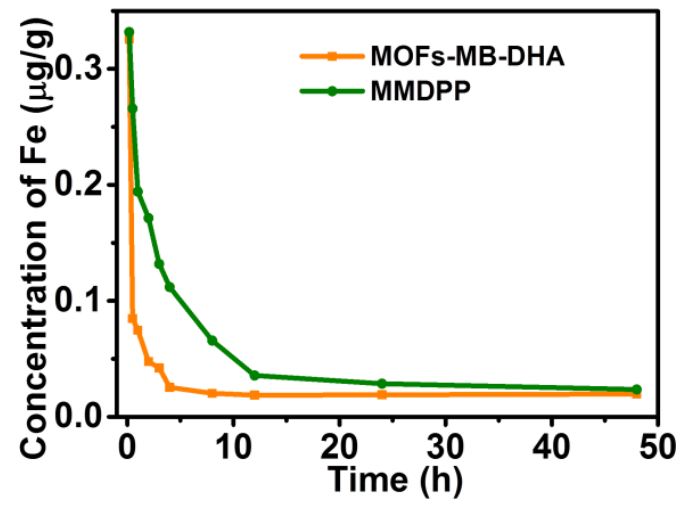

Figure S20. The content of Fe ion in the blood of mice at different time points after intravenous injection of MOFs-MB-DHA or MMDPP. 